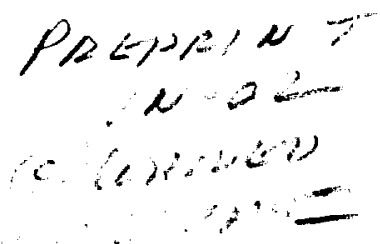

AIAA 2000-0757

Wake Vortex Transport and Decay in Ground Effect: Vortex Linking with the Ground

Fred $\mathrm{H}$. Proctor and David W. Hamilton

NASA Langley Research Center

Hampton, VA

Jongil Han

North Carolina State University

Raleigh, NC

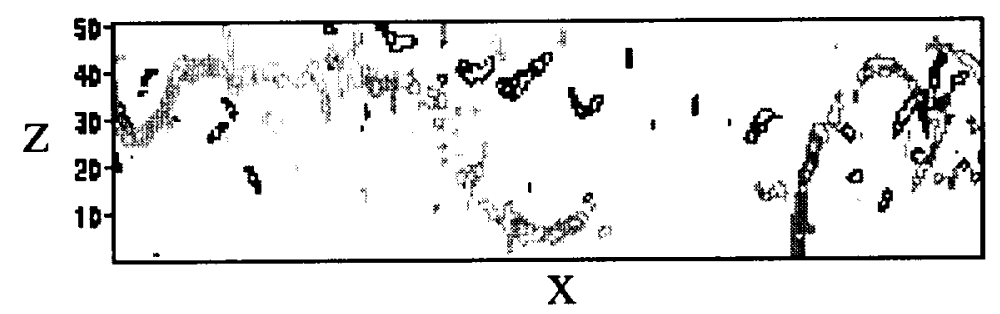

38th Aerospace Sciences Meeting \& Exhibit

January 10-13, 2000 / Reno, NV

For permission to copy or republish, contact the American Institute of Aeronautics and Astronautics 1801 Alexander Bell Drive, Suite 500, Reston, Virginia 20191-4344 
AIAA-2000-0757

\title{
Wake Vortex Transport and Decay in Ground Effect: Vortex Linking with the Ground
}

\author{
Fred $\mathrm{H}$. Proctor* and David W. Hamilton ${ }^{\dagger}$ \\ NASA Langley Research Center \\ Hampton, Virginia \\ Jongil Han ${ }^{\ddagger}$ \\ North Carolina State University \\ Raleigh, NC
}

\begin{abstract}
Numerical simulations are carried our with a threedimensional Large-Eddy Simulation (LES) model to explore the sensitivity of vortex decay and transport in ground effect (IGE). The vortex decay rates are found to be strongly enhanced following maximum descent into ground effect. The nondimensional deray rate is found to be insensitive to the initial values of circulation, height. and vortex separation. The infonmation gained from these simulations is used to construct a simple decay relationship. This relationship compares well with observed data from an IGE rase study. Similarly, a relationship for lateral drift due to ground effect is constructed from the LES data. In the second part of this paper, vortex linking with the ground is investigated. Our numerical simulations of wake vortices for IGE show that a vortex may link with its inage beneath the ground, if the intensity of the ambient urbulence is moderate to high. This linking with the ground (which is observed in real cases) gives the appearance of a vortex tube that bends to
\end{abstract}

*Research Scientist, Airborne Systems Competency, AIAA member

${ }^{\dagger}$ Research Scientist, Airborne Systems Competency

${ }^{\ddagger}$ Research Scientist, Department of Marine, Earth, and Atmospheric Sciences. AlAA member

Copyright $(2) 2000$ by the American Institute of Aeronautics and Astronautics. Inc. No copyright is asserted in the United States under Title 17, U.S. Code. The Government has a royalty-free license to exercise all rights under the copyright claimed herein for Government purposes. All other rights are reserved by the copyright owner. become vertically oriented and which terminates at the ground. From the simulations condurted, the linking time for IGE appears to be similar to the linking time for vortices in the free atmosphere: i.e. a function of ambient turbulence intensity.

\begin{tabular}{|c|c|}
\hline & Nomenclature \\
\hline$B$ & aircraft wing span \\
\hline$b_{0}$ & initial vortex separation $-\pi B / 4$ \\
\hline$g$ & acceleration due to gravity \\
\hline$L_{x}, L_{y}$ & domain length in $\mathrm{x}$ and $\mathrm{y}$ directions, respectively \\
\hline$L_{z}$ & domain depth \\
\hline$M$ & mass of generating aircraft \\
\hline$r$ & radius from vortex center \\
\hline$r_{r}$ & radius of peak tangential velocity \\
\hline$t$ & time coordinate \\
\hline$T$ & nondimensional time $-\imath V_{0} / b_{0}$ \\
\hline$T_{t ;}$ & $\begin{array}{l}T \text { at } z=z_{\min } \\
\text { airspeed of generating aircraft }\end{array}$ \\
\hline$v_{\mathrm{o}}$ & initial vortex descent velocity $-\Gamma_{0} /\left(2 \pi b_{0}\right)$ \\
\hline$x$ & horizontal coordinate along flight path \\
\hline$y$ & horizontal coordinate lateral to fight path \\
\hline$Y$ & nondimensional lateral coordinate $-y / b_{c}$ \\
\hline$z$ & vertical coordinate \\
\hline$z_{\min }$ & minimum altitude achieved during vortex descent \\
\hline $\begin{array}{l}Z_{i} \\
\Gamma\end{array}$ & $\begin{array}{l}\text { initial height above ground of wake vortex } \\
\text { vortex circulation }\end{array}$ \\
\hline$\Gamma_{0}$ & initial vortex circulation $-M g /\left(b_{\mathrm{s}} \rho V_{\mathrm{a}}\right)$ \\
\hline$\rho$ & air density \\
\hline$\epsilon$ & ambient turbulence (eddy) dissipation rate \\
\hline$\zeta_{\mathrm{x}}$ & $x$-component of vorticity \\
\hline$v$ & kinematic viscosity \\
\hline$\eta$ & nondimensional eddy dissipation $-\left(\epsilon b_{0}\right)^{\prime}$ \\
\hline
\end{tabular}




\section{Introduction}

Aircraft wake vortices are formed when vorticity generated by lift rolls up into two primary trailing vortices. The two vortices have a characteristic initial separation, $\mathbf{b}_{0}$, which is proportional to the wingspan of the generating aircraft. The wake vortices are considered to be "in ground effect" (IGE) when located within one initial separation of the ground (i.e., $z<b_{0}$ ) ${ }^{\prime}$ The prediction for the location and intensity of these vortices is important for systems that are to manage safe aircraft spacings.

The complex influence of the ground on wake vortex behavior has been the focus of numerous investigations, including the observations by Harvey and Perry, Hallock, ${ }^{3}$ Kopp, ${ }^{4}$ Hallock and Burnham, ${ }^{9}$ Burhnam and Hallock, ${ }^{\circ}$ and Rudis et al., ${ }^{7}$ as well as the numerical studies by Schiling, ${ }^{8}$ Robins and Delisi, ${ }^{9}$ Zheng and Ash, ${ }^{10}$ Corjon and Poinsot, ${ }^{1}$ Corjon and Stoessel, ${ }^{12}$ and Proctor and Han. ${ }^{13}$ Qualitative understanding of basic vortex transport mechanisms for IGE is known, but quantitative relationships are needed. Wake vortex decay is known to be enhanced by proximity to the ground, but much is to be learned about this behavior. ${ }^{6}$

Three-dimensional instability of wake vortices near the ground is largely unexplored. Lateral linking of the vortex pair associated with Crow instability ${ }^{14}$ should be less likely due to the increased separation forced by the ground's presence. Ground linking -- the linking of a vortex with its image vortex beneath the ground plane -. has been observed, ${ }^{15}$ but thought to be rare. ${ }^{16}$ Linking instabilities can increase the uncertainty of a vortex's position and may affect the vortex decay rate as well.

Semi-empirical vortex prediction algorithms have been developed by Robins et $a l^{\prime}$ ' that include the ground's influence on lateral separation and vortex rebound. Similar algorithms ${ }^{17}$ have been incorporated within NASA's Aircraft VOrtex Spacing System (AVOSS). 18.19.20 Improved accuracy of these prediction algorithms depend on the better understanding of vortex transport and decay near the ground. Numerical experiments with a Large Eddy Simulation (LES) model are being conducted in order to provide guidance for the enhancement of these prediction algorithms. ${ }^{21}$

This paper is a continuation of the three-dimensional LES study described in Proctor and Han, ${ }^{13}$ with further focus on vortex decay within IGE. In Proctor and Han, we investigated the sensitivity over a wide range of ambient turbulence levels of a wake vortex for a landing $L-1011$ that was observed on 26 September 1997 at Dallas-Fort Worth (DFW) airport. ${ }^{22}$ The simulations showed only a weak sensitivity to the level of ambient turbulence, and were in very-good agreement with Lidarderived observations. The results indicated that enhanced decay from ground effect begins a few seconds after the vortices descend to their minimum height. with vortex decay prior to these times being primarily influenced by stratification and ambient turbulence. The study also found that vertical oscillations associated with vortex rebound were less prominent for higher levels of ambient turbulence, since the secondary vortices were more likely to be diminished by the stronger turbulence. Also addressed was the influence of ground stress on wake vortex transport. The study determined that viscous stress reduced the velocity near the ground and acted to decouple the primary vortices from their sub-ground images. If ground stress was ignored, unrealistic divergence of the vortex pairs ensued. The study, did not address Crow instability, or similar linking instabilities.

Table 1. Salient Characteristics of TASS

Primitive equation / non-Boussinesq equation set Timedependent, nonhydrostatic, compressible.

Meleorological framework with option for either threedimensional or two-dimensional simulations.

Large Eddy Simulation model with 1st-order subgrid scale turbulence closure - Grid-scale turbulence explicitly computed, while effects of subgrid-scale turbulence modeled by Smagorinsky model with modifications for stratification and flow rotation.

Ground stress based on Monin-Obukhov Similarity theory.

Optional boundary conditions - open conditions utilizes mass-conservative, nonreflective radiation boundary scheme.

Explicit numerical schemes, quadratic conservative, time-split compressible- accurate as well as highly efficient, and essentially free of numerical diffusion. Space derivatives computed on Arakawa C-grid staggered mesh with $4^{\text {th }}$-order accuracy for convective terms.

Prognostic equations for vapor and atmospheric water substance (e.0. cloud droplets, rain, snow, hail, ice crystals). Large set of microphysical-parametenization models.

Model applicable to mesoy and microscale atmospheric phenomenon. Initialization modules for simulation of convective storms, microbursts, atmospheric boundary layers, turbulence, and aincraft wake vortices. 
since the assumed domain had a limited axial depth. In the present paper, we address the sensitivity of vortex decay for IGE, by conducting LES of wake vortices with different initial heights, separations and circulations. The results are normalized and a simple analytical representation for IGE decay is given. Similarly, a simple model based on the LES results is proposed for lateral drift due to ground effect. In the second part of this paper, we examine the occurrence of ground linking and its sensitivity to ambient turbulence.

\section{The Model and Initial Conditions}

The numerical model used in this study is a threedimensional LES model called the Terminal Area Simulation System ${ }^{23}$ (TASS), which has been adapted for simulation of wake vortex interaction with the atmosphere. ${ }^{24}$ The numerical model used in this study differs from that in many previous investigations, in that: 1) time-dependent computations are carried out in threedimensional space, 2) the formulation is essentially free of numerical diffusion, ${ }^{25}$ ) the computations are LES and at high Reynolds number, 4) the experiments are initialized with realistic turbulence fields, and 5) realistic surfacestress and subgrid turbulence-closure formulations are assumed. Salient characteristics of TASS are listed in table 1.

\section{Model Equations}

The TASS model contains a prognostic equation set for momentum, temperature and pressure, and employs a compressible time-split formulation. Omitting moisture and coriolis terms (which are not used in these simulations), the TASS equation set in standard tensor notation is as follows:

Momentum

$$
\begin{aligned}
& \frac{\partial_{u_{i}}}{\partial t}+\frac{H}{\rho_{o}} \frac{\partial p}{\partial x_{i}}=-\frac{\partial_{u_{i} u_{j}}}{\partial x_{j}}+u_{i} \frac{\partial_{u_{j}}}{\partial x_{j}}+g(H-J) \delta_{i 3} \\
& +\frac{1}{\rho_{o}} \frac{\partial}{\partial x_{j}} \rho_{o} K_{M}\left[\frac{\partial_{u_{i}}}{\partial x_{j}}+\frac{\partial_{u_{j}}}{\partial x_{i}} \cdot \frac{2}{3} \frac{\partial u_{k}}{\partial x_{k}} \delta_{i j}\right)
\end{aligned}
$$

Buoyancy Term:

$$
H=\left(\frac{\theta}{\theta_{o}}-\frac{p C_{v}}{P_{o} C_{p}}\right]
$$

Pressure Deviation:

$$
\begin{aligned}
& \text { Pressure Deviation: } \\
& \frac{\partial p}{\partial t}+\frac{C_{p} P}{C_{V}} \frac{\partial_{u j}}{\partial x_{j}}=\rho_{o} g u_{j} \delta_{j 3}
\end{aligned}
$$

Thernodynamic Equation (Potential Temperature):

$$
\begin{aligned}
\frac{\partial \theta}{\partial t}= & -\frac{1}{\rho_{o}} \frac{\partial \theta \rho_{o} u_{j}}{\partial x_{j}}+\frac{\theta}{\rho_{o}} \frac{\partial \rho_{o} u_{j}}{\partial x_{j}} \\
& +\frac{l}{\rho_{o}} \frac{\partial}{\partial x_{j}}\left[\rho_{o} K_{H} \frac{\partial \theta}{\partial x_{j}}\right]
\end{aligned}
$$

with the Potential Temperature defined as

$$
\theta=\tau\left(\frac{P_{o w}}{P}\right) \frac{K_{d}}{c_{p}}
$$

In the above equations, $u_{i}$ is the tensor component of velocity, $t$ is time, $p$ is deviation from atmospheric pressure $P, \tau$ is atmospheric temperature, $\rho$ is the air density, $C_{p}$ and $C_{v}$ are the specific heats of air at constant pressure and volume, $g$ is the earth's gravitational acceleration, $R_{d}$ is the gas constant for dry air, $P_{o o}$ is a constant equivalent to 1000 millibars $\left(10^{5}\right.$ pascals) of pressure. Environmental state variables, e.g., $\rho_{o}, P_{o}$ and $\theta_{o}$, are defined from the initial input sounding and are functions of height only.

A modified Smagorinsky first-order closure is used for the subgrid eddy viscosity as:

$$
\begin{aligned}
K_{M}= & l_{s}^{2} \sqrt{\frac{\partial_{u_{i}}}{\partial x_{j}}\left(\frac{\partial_{u_{i}}}{\partial x_{j}}+\frac{\partial_{u_{j}}}{\partial x_{i}}\right)-\frac{2}{3}\left(\frac{\partial_{u_{k}}}{\partial x_{k}}\right)^{2}} \\
& x \sqrt{1-\alpha_{1} R i_{s}-\alpha_{2} R i_{r}}
\end{aligned}
$$

The subgrid eddy viscosity for momentum, $\boldsymbol{K}_{M}$. is modified by the Richardson numbers, for stratification, $R i_{s}$, and for flow rotation, $R i_{\text {r, with }} \alpha_{1}=3$ and $\alpha_{2}=1.5$.

The subgrid length scale, $l_{s}$, is determined from the grid volume and is matched to the appropriate length scale close to the ground where the flow is under-resolved. That is:

$$
l_{s}=\left[\begin{array}{cl}
\alpha \Delta & z \geq \alpha \Delta / k \\
\frac{\alpha \Delta\left(l+(\alpha \Delta / k z)^{m-1}\right)}{1+(\alpha \Delta / k z)^{m}} & \alpha \Delta / k>z>\Delta z / 2 \\
k z & z \leq \Delta z / 2
\end{array}\right.
$$

where $k$ is von Karman's constant, and where $m$ and $\alpha$ are invariant constants with values defined as $m=3$ and $\alpha=$ 0.15 . The filter width is based on the minimal resolvable scale:

$$
\Delta=|2 \Delta x 2 \Delta y 2 \Delta z|^{1 / 3}
$$


where $\Delta x, \Delta y$, and $\Delta z$ are the numerical grid sizes in the respective $x, y, z$ direction.

The ground boundary is impermeable with nonslip velocity specifications. The surface stress due to the ground is determined locally from the wind speed, surface roughness, and the local thermal stratification. Details of the surface formulation are in the appendix of reference [13].

Turbulence Initialization

Prior to vortex initialization, an initial field of resolved-scale turbulence is allowed to develop under an artificial external forcing at low wavenumbers. ${ }^{26}$ The approach is similar to that in recent studies with TASS, where wake vortex decay and the development of Crow instability is examined within a Kolmogorov ${ }^{27}$ spectrum of homogeneous turbulence (Han et al. ${ }^{28.29}$ ). The method, however, is slightly different for the present study, due to the inclusion of the ground. Since periodic boundary conditions are assumed only at the horizontal boundaries, the turbulence forcing is applied only to horizontal velocity over each horizontal plane. Nevertheless, the influence of the horizontal two-dimensional forcing spreads quickly to the vertical direction as well as to the vertical velocity through the mass continuity.

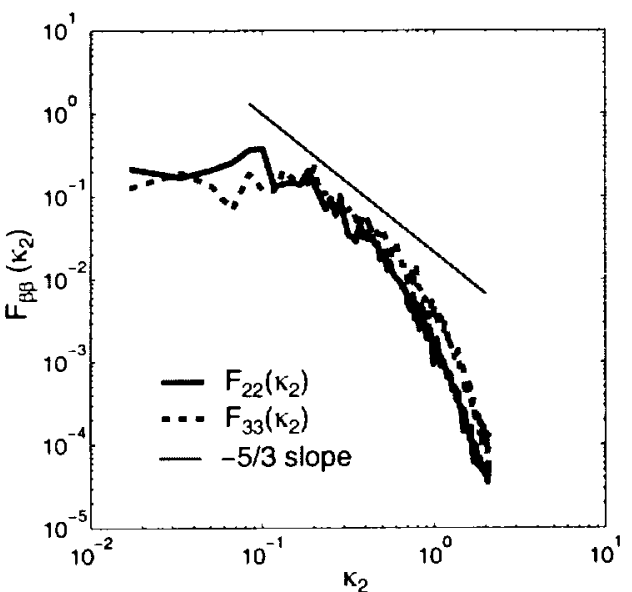

Figure 1. Turbulence energy spectrum at $:=14 \mathrm{~m}$ before vortex injection. Here, subscripts 2 and 3 denote crossflow and vertical directions, respectively.

Because the TASS code uses a finite difference numerical scheme, the forcing is achieved by performing, first, a two-dimensional fast Fourier transform (FFT) at every large time step, then adding a constant amplitude to all the modes with integer wavenumbers whose magnitude is less than 3.0, and finally, performing an inverse FFT back to the physical space. At the same time, the horizontal domain average temperature and velocity fields are forced to maintain their initial vertical profiles by subtracting the difference every time step. Due to subgrid dissipation, the simulation can reach a statistically steady state, in the sense that the mean turbulence kinetic energy oscillates in time around a constant value.

Figure 1 shows a one-dimensional energy spectrum with a $-5 / 3$ slope of Kolmogorov's ${ }^{27}$ spectrum when the turbulent flow field has achieved a statistically steady state. Figure 1 indicates that our approach can produce a well-developed turbulent flow field that possesses Kolmogorov's inertial subrange. The eddy dissipation rate $\varepsilon$ is estimated from the well-known technique of fitting Kolmogorov's theoretical spectrum in the inertial subrange to the simulated spectra

\section{Vortex Initialization}

The initial wake vortex field is specified with a simple vortex system that is representative of the post rollup, wake-vortex velocity field. The vortex system is initialized with the superposition of two counter-rotating vortices that have no initial variation in the axial direction The tangential velocity, $V$, associated with each vortex, is determined from:

$V(r)=\frac{\Gamma_{\infty}}{2 \pi r}\left[1-\operatorname{Exp}\left(-10\left(\frac{r}{B}\right)^{0.75} /\right]\right.$

where, $r$ is the radius from the center of the vortex. $\Gamma_{x}$ the circulation at $r \gg r_{c}$, and $B$ is the span of the generating aircraft. The above formula is based on Lidar observations of wake vortices measured early in their evolution. The model is applied only at $r>r_{c}$ and is matched with a Lamb model profile for $r<r_{c}$. The values assumed for initial vortex separation and circulation are derived from an aircraft's weight, span. and airspeed according to conventional formula based on elliptical loading; i.e., $b_{n}=\pi B / 4$, and $\Gamma_{\infty}=4 M g /\left(\pi B \rho V_{n}\right)$ Appropriate vortex image conditions are applied to the initial wake field to ensure consistency and mass continuity at the TASS model boundaries.

Except when otherwise noted, the initial vortex parameters (table 2) are taken from the observed aircraft parameters for the L-1011, as was used in Proctor and Han. ${ }^{13}$

All simulations are conducted with dimensional variables and assume turbulent flow with a rotational Reynolds number $\left(\Gamma_{v} / v\right)$ of $\sim 10^{7}$. 
Table 2. Initial vortex parameters (baseline).

\begin{tabular}{ll}
\hline Parameter & Value \\
\hline Vortex spacing $\left(b_{\mathrm{v}}\right)$ & $37 \mathrm{~m}$ \\
Generating height $\left(Z_{\mathrm{i}}\right)$ & $16 \mathrm{~m} \quad\left(0.432 b_{\mathrm{v}}\right)$ \\
Vortex circulation $\left(\Gamma_{\mathrm{v}}\right)$ & $390 \mathrm{~m}^{2} \mathrm{~s}^{-1}$ \\
Vortex core radius & $3 \mathrm{~m} \quad\left(b_{\mathrm{v}} / 12.3\right)$ \\
\hline
\end{tabular}

Model Domain Parameters

Two domain sizes are used for the experiments in this paper. A short domain, which has been truncated in the $x$-direction $\left(L_{x}, L_{y}, L_{z}=81 \mathrm{~m}, 370 \mathrm{~m}, 81 \mathrm{~m}\right)$, and a long domain $\left(L_{x}, L_{y}, L_{z}=4.51 \mathrm{~m}, 3.37 \mathrm{~m}, 81 \mathrm{~m}\right)$. For an assumed initial vortex separation of $b_{o}=37 \mathrm{~m}$, this would translate into: $(2.2 \times 10 \times 2.2) b_{o}$ for the short domain and $(12.2 \times 9.1 \times 2.2) b_{o}$, for the long domain. Both long and short domains are resolved by the grid sizes listed in table 3.

Table 3. Grid Resolution

\begin{tabular}{ll}
\hline Parameter & Value \\
\hline Vertical resolution $(\Delta z)$ & $1.5 \mathrm{~m}$ \\
Lateral resolution $(\Delta y)$ & $1.5 \mathrm{~m}$ \\
Axial resolution $(\Delta x)$ & $2.0 \mathrm{~m}$ \\
\hline
\end{tabular}

The advantage of the short domain is that it allows economical calculations of vortex behavior and simplifies the analysis of results. The longer domain, however, is less likely to hinder three-dimensional linking instabilities. A comparison of results from the two domain sizes is shown in the appendix.

Determination of Vortex Position and Circulation

Based on the vortex position in each crossflow plain, circulation is computed for each crossflow plane according to:

$\Gamma_{r}(x)=\iint_{r} \zeta_{x} d y d z$

The circulation is not computed for any plane where the vorticity vector is beyond $30^{\circ}$ of the $x$-axis.

A $.5-15$ meter averaged-circulation, is computed according to:

$\bar{\Gamma}^{a, b}=\frac{\int_{a}^{b} \Gamma_{r} d r}{\int_{a}^{b} d r}$

where $a=5 \mathrm{~m}$, and $b=15 \mathrm{~m}$. The $5.15 \mathrm{~m}$ average circulation is chosen to characterize the intensity of the vortex, since it quantifies the hazard faced by an encountering aircraft ${ }^{30,31}$
A mean average circulation is reported for each time interval by computing a mean of the circulations from each crossflow plane:

$$
\overline{\Gamma_{\bar{x}}} a, b=\frac{I}{N} \sum_{i}^{N} \bar{\Gamma}_{i} a, b
$$

Similarly, a mean yortex position is reported for each time interval by averaging the positions from each crossflow plane.

\section{IGE Sensitivity}

Three sets of experiments are conducted in order to determine the sensitivity of vortex decay within IGE. The short domain is assumed which will inhibit the occurrence of linking instabilities. All of the simulations assume neutral stratification, no mean ambient winds, and an ambient turbulence dissipation rate of $\epsilon=9.6 \times 10^{-5} \mathrm{~m}^{2}$ $s^{-3}$. Other initial conditions for the simulations are listed in tables 2 and 3 .

\section{Sensitivity to Initialization Height}

Five experiments are conducted for a vortex initialization height $\left(Z_{i}\right)$ of: $0.32 b_{m}, 0.5 b_{o}, 0.65 b_{c m} 0.84$ $b_{o,}$ and $1.0 b_{o}$. Comparisons of the experiments are shown in Fig. 2 as function of nondimensional time. Note that the time coordinate for each experiment is offset by the time of maximum descent into ground effect; i.e., by $T_{i}=T\left(\Sigma_{\min }\right)$. The curves for normalized $5-1.5 \mathrm{~m}$ average circulation, as well as the normalized lateral positions tend to collapse, once the of fset time $T_{6}$ is accounted for. As also shown in our previous study, ${ }^{13}$ vortex decay is significantly enhanced following maximum penetration into IGE. In addition, these results show the decay rate to be more or less independent of the vortex initial height, with the enhanced level of decay beginning roughly 0.25 nondimensional time units following $T_{G}$;

A formula for the decay of average circulation can be obtained from these results as

$\frac{\Gamma}{\Gamma_{o o}}=\operatorname{Exp}\left(\frac{-2\left(T-T_{o r o}\right)^{2 / 3}}{5}\right)$

where $T_{n o}=T_{i i}+0.25$ (i.e., 0.25 nondimensional time units following the time of maximum penetration into ground effect), and $\Gamma_{s w}$ is the circulation at nondimensional time $T_{n o}$. The above formula is independent of ambient turbulence and can only be applied for $T>T_{i}+0.25$.

Differentiating the above gives the rate of decay as: 


$$
\frac{I}{\Gamma} \frac{d \Gamma}{d T}=-\frac{4}{15\left(T-T_{o o}\right)^{1 / 3}} \operatorname{Exp}\left(\frac{-2\left(T-T_{o o}\right)^{2 / 3}}{5}\right)
$$

A plot of the curve from $E q . I$ is shown also in Fig. $2 c$ and agrees very well with the LES results.

An empirical relationship for lateral spread within IGE can be determined from the LES results as well. The vortices, which start at a lateral distance from the flight path of $y= \pm 1 / 2 b_{w}$, diverge lateral with time due to the influence of the ground. Figure $2 \mathrm{~b}$ indicates (in the absence of crosswind) that the vortices asymptote toward $y=2 b_{o}$; approaching a maximum separation of about $4 b_{o}$. Based on these results, an empirical relationship for vortex drift due to ground effect is:

$$
Y=1.38 .5\left(T-T_{i}\right)^{m .227} \text { for } T>T_{i}+0.25
$$

where $Y$ is the normalized lateral position from the flight path. The vortex separation, $\mathrm{b}=2 \mathrm{Yb}$ o also can be predicted for IGE with Eq. 2. A plot of the curve from Eq. 2 is compared with LES data in Fig. $2 b$.

The lateral drift rate can be determined by differentiating (2) giving:

$V_{G}=\frac{d Y}{d T}=0.3173\left(T-T_{G}\right)^{-0.773}$ for $T>T_{G}+0.25$

where $V_{G i}$ is the nondimensional velocity for lateral drift due to ground effect (this velocity can be made dimensional by multiplying by the initial vortex sink rate, $\left.V_{o}\right)$.

Sensitivity to Initial Vortex Spacing

In order to further evaluate the sensitivity of our results and test the validity of $E q$. 1 , three additional experiments are conducted, which assume different initial vortex spacings $\left(b_{0}\right)$ as listed in table 4 . An initial circulation of $400 \mathrm{~m}^{2} \mathrm{~s}^{-1}$ and an initial height of $Z_{i}=0.432$ $b_{a}$ is assumed for each experiment. Values for $Z_{i}$ and nondimensional turbulence dissipation are slightly different in these experiments due to the variation in $b_{0}$.

Table 4. Initial vortex separation $\left(b_{o}\right)$ and corresponding initial altitude $\left(Z_{i}\right)$ and dimensionless turbulence intensity $(\eta)$ for sensitivity to initial vortex spacing cases.

\begin{tabular}{ccc}
\hline$b_{0}(m)$ & $Z_{i}(m)$ & $\eta$ \\
\hline 30 & 13 & 0.0671 \\
37 & 16 & 0.0897 \\
49 & 21.2 & 0.1291 \\
\hline
\end{tabular}

IGE Sensitivity Test for Varying Vortex Heights ( $\eta=0.091$ )
Vortex Vertical Position History (Port)

(A)

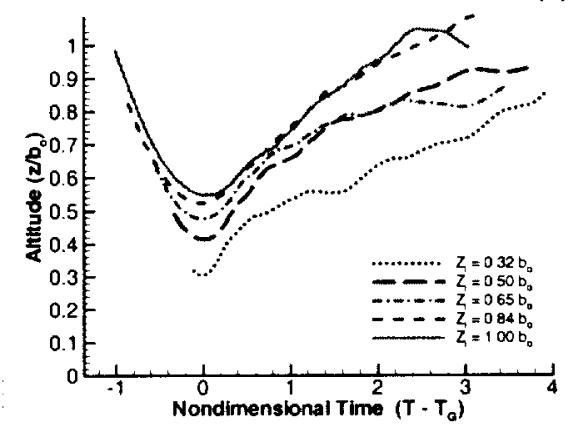
IGE Sensitivity Test for Varying Vortex Heights ( $\eta=0.091$ )
Vortex Lateral Position History (Port)

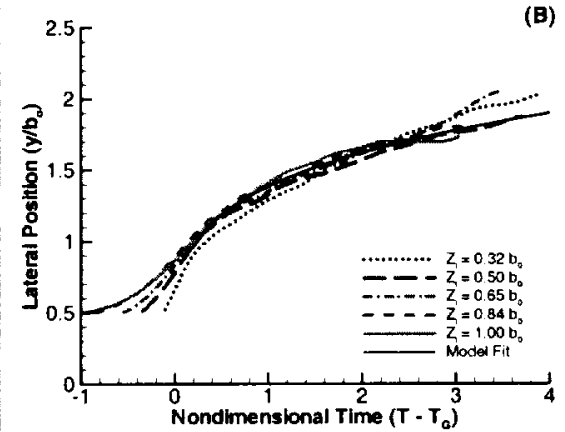

IGE Sensitivity Test for Varying Vortex Heights ( $\eta=0.091$ ) Vortex 5-15 m Averaged Circulation History (Port)

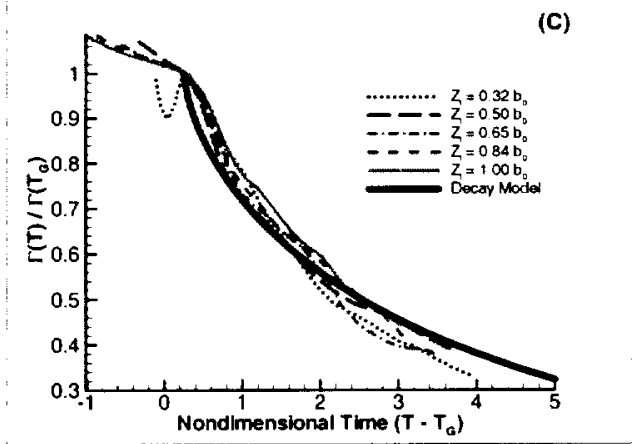

Figure 2. Sensitivity o vortex initiation height. Nondimensional height (a), nondimensional lateral position (b), and nondimensional $5.15 \mathrm{~m}$ average circulation (c) vs nondimensional time. "Deray model" in (C) from Eq.l and "Curne fit" in (B) from Eq.2. 


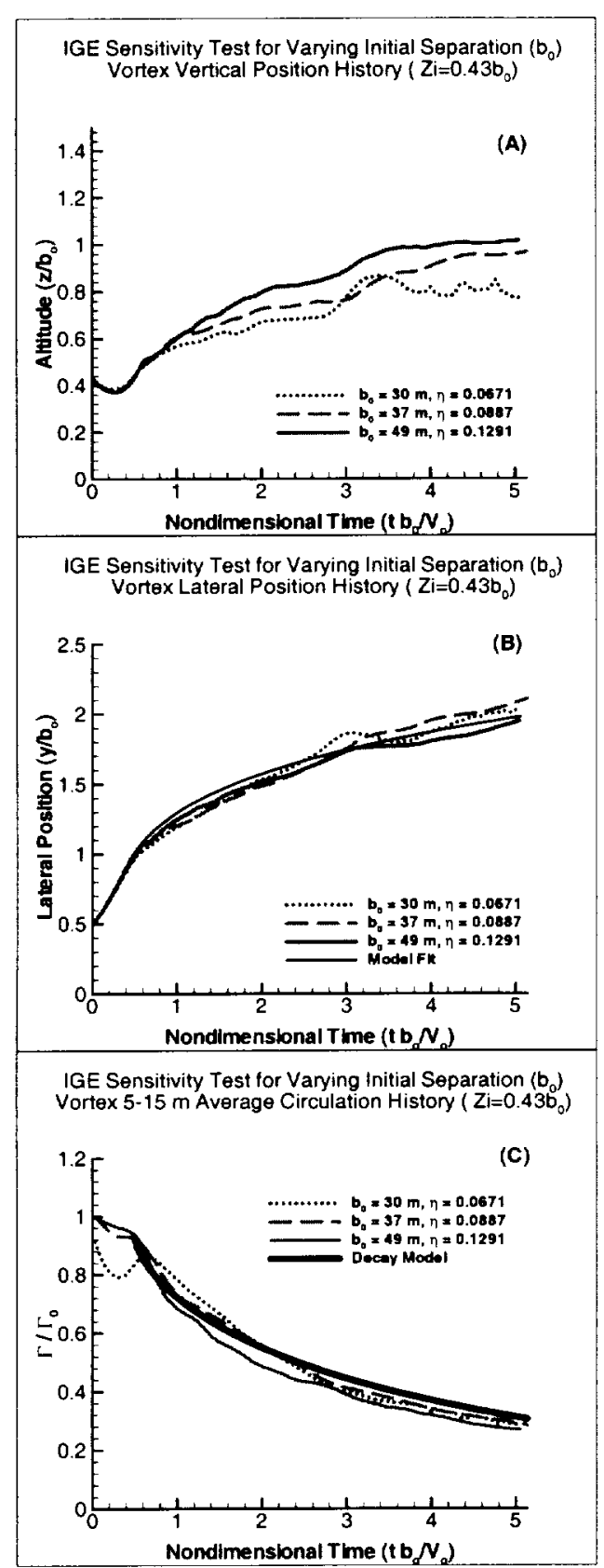

Figure 3. Same as Fig. 2, but for sensitivity to initial vortex spacing.
IGE Sensitivity Test for Varying Initial Circulation Vortex Vertical Position History

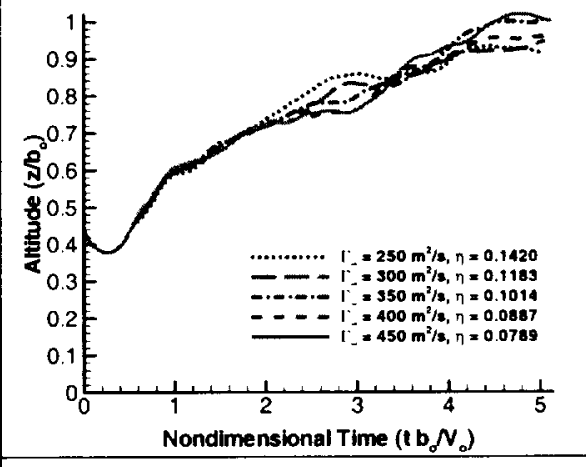

IGE Sensitivity Test for Varying Initial Circulation Vortex Lateral Position History

(B)

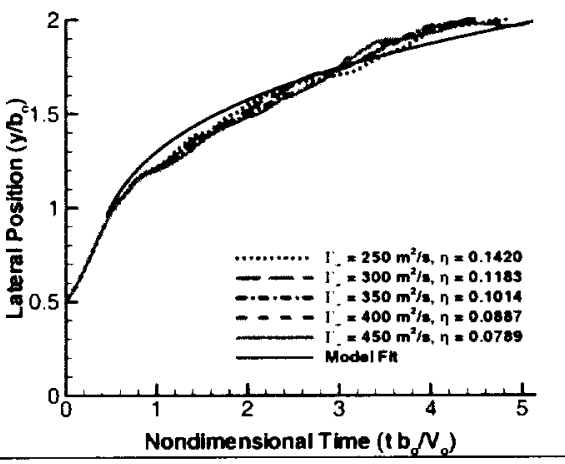

IGE Sensitivity Test for Varying Initial Circulation Vortex 5-15 m Average Circulation History

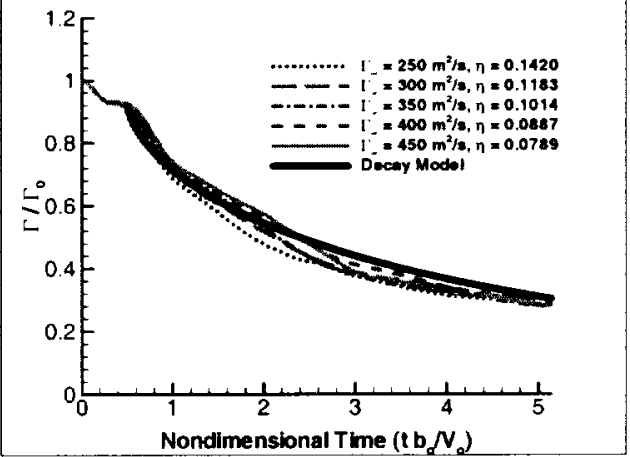

Figure 4. Same as Fig.2, but for sensitivify to initial vortex circulation.

7

American Institute of Aeronautics and Astronautics 
The results presented in Fig. 3 show that the curves again collapse to a similar value when normalized, and that the proposed formulas for IGE decay and lateral drift are in excellent agreement with the LES results. Enhanced levels of decay due to IGE begin at $T=0.5$ (which is $T_{i}+0.25$ ) for all three cases.

\section{Sensitivity to Initial Circulation}

In the third set of experiments, five simulations with different initial circulations are conducted (table 5). All else is assumed equal, with $\eta$ being different due to its dependency on $\Gamma_{o}$.

Again, the curves collapse with a tight spread (Fig. 4 ), and the proposed IGE decay and drift formulas are in excellent agreement with LES results

Table 5. Initial circulation $\left(\Gamma_{0}\right)$ and corresponding dimensionless turbulence intensity for $\Gamma_{0}$ sensitivity cases.

\begin{tabular}{cc} 
dimensionless turbulence intensity for $\Gamma_{0}$ sensitivity cases. \\
\hline$\Gamma_{0}\left(m^{2} s^{-1}\right)$ & $\eta$ \\
250 & 0.1420 \\
300 & 0.1183 \\
350 & 0.1014 \\
400 & 0.0887 \\
450 & 0.0789 \\
\hline
\end{tabular}

\section{Comparison with Observed Case.}

In this section, the new formula for IGE decay (Eq. 1) is compared with the LES simulation for a landing $L-1011$, which was observed at 20:09 UTC on 26 September 1997 at Dallas-Fort Worth (DFW) airport. The input sounding for temperature and crosswind is shown in Fig. 5. The simulation is conducted with the short domain and with the parameters listed in tables 2 and 3 . The simulation includes an ambient turbulence field with, $\epsilon=1.654 \times 10^{-3} \mathrm{~m}^{2} \mathrm{~s}^{-3}(\mathrm{n}=0.2349)$, which is very close to the ambient value observed at $z=40 \mathrm{~m}$. The average circulation from both the port and starboard vortices is show in Fig. 6 . The slightly faster decay for the starboard vortex may be due to the opposite sign vorticity of the ambient crosswind. Also included in Fig. 6 for comparison, is measured Lidar data for the port vortex. The LES results and Lidar data show reasonable agreement with the circulation decay given by Eq. 1 .

\section{Ground Linking Experiments}

In the free atmosphere, the linking process begins as the counter-rotating circulations of a vortex pair connect. producing crude vortex rings. The time at which this linking takes place is a known function of the turbulence intensity, or specifically, the eddy dissipation rate ${ }^{28,32,33}$ The goal of the experiments in this section is to examine ground linking and any sensitivity that it may have to the level of ambient turbulence. All of the experiments assume the long domain so as to permit linking instabilities. The experiments also assume the parameters listed in tables 2 and 3 , as well as the initial sounding in Fig. 5. The nondimensional turbulence strengths, $\eta$, used in these experiments, range from 0.233 to 0.75 . These values represent a typical range between moderate to very turbulent atmospheric boundary layers.
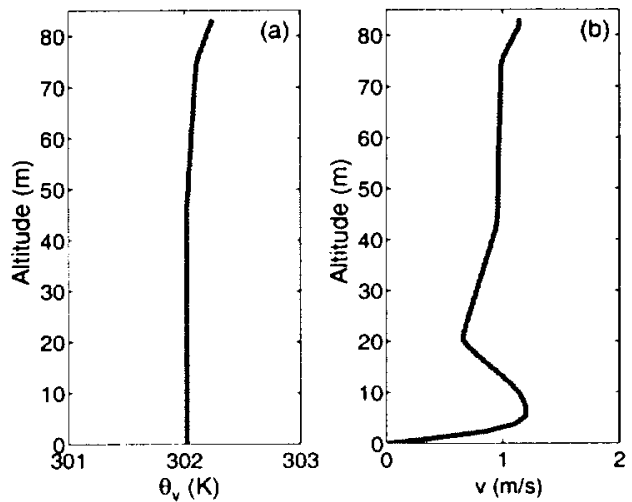

Figure 5. Initial profile of a) ambient potential temperature and b) crosswind for DFW, 26 September 1997, 20:09 UTC.

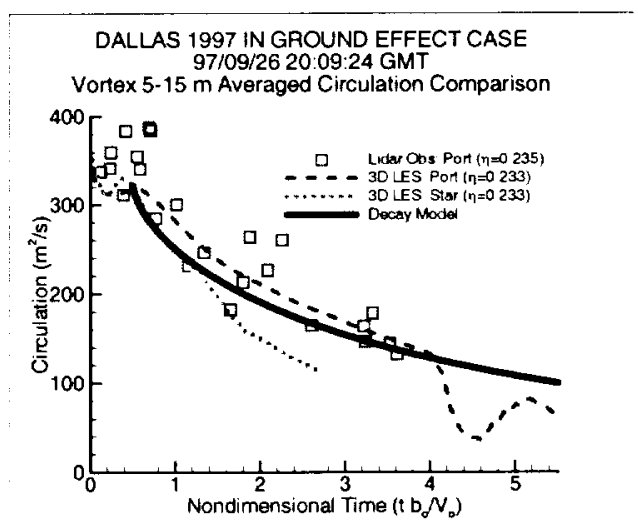

Figure 6. Average circulation vis nondimensional time for observed DFW case. Comparison of LES, Lidar, and IGE decay model (Eq. I). 


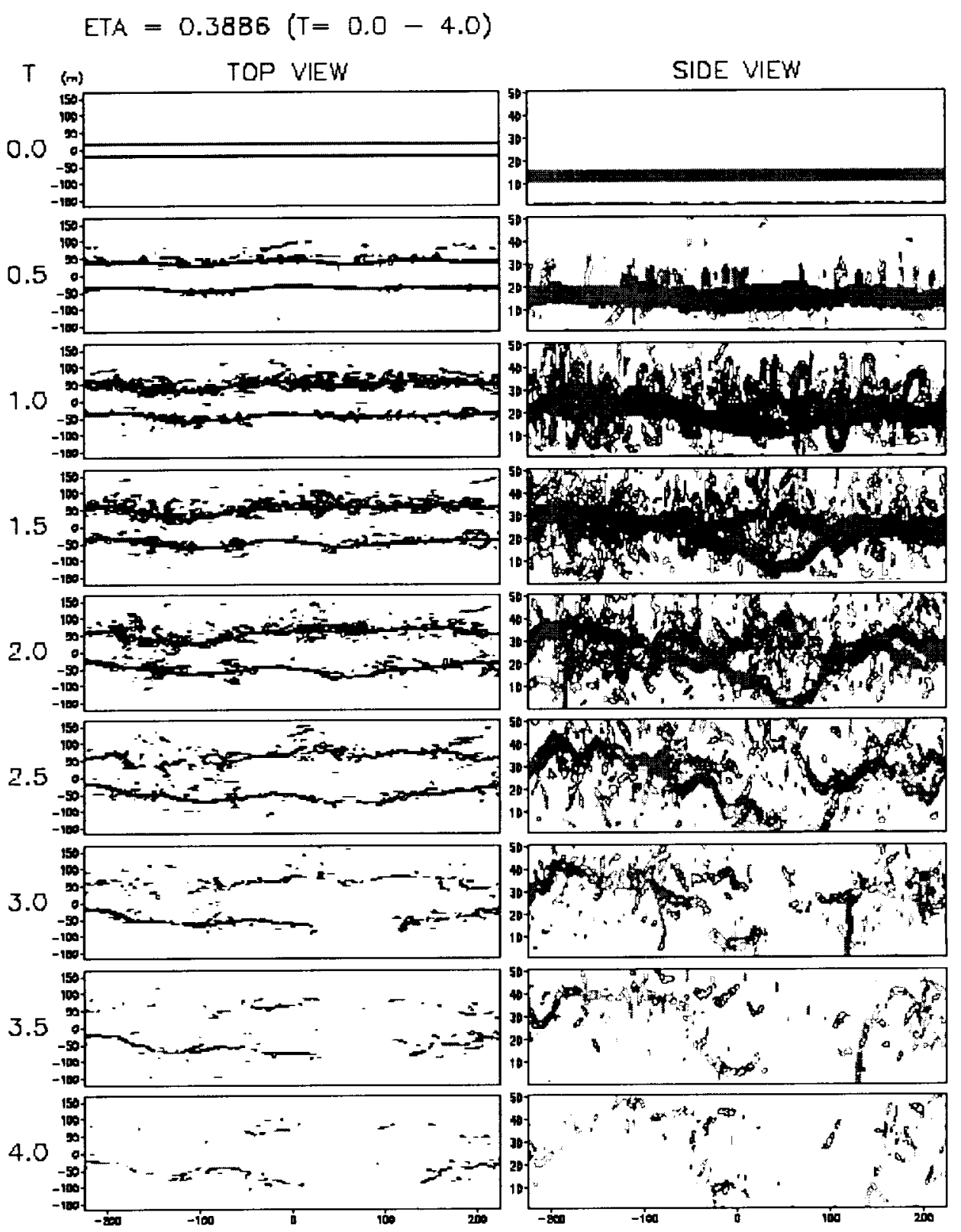

Figure 7. Time evolution within IGE of wake vortex pair for $\eta=0.388$. Top ( $x, y)$ and side ( $x$, z) view of wake vortices at increments 0.5 nondimensional time uniss. 

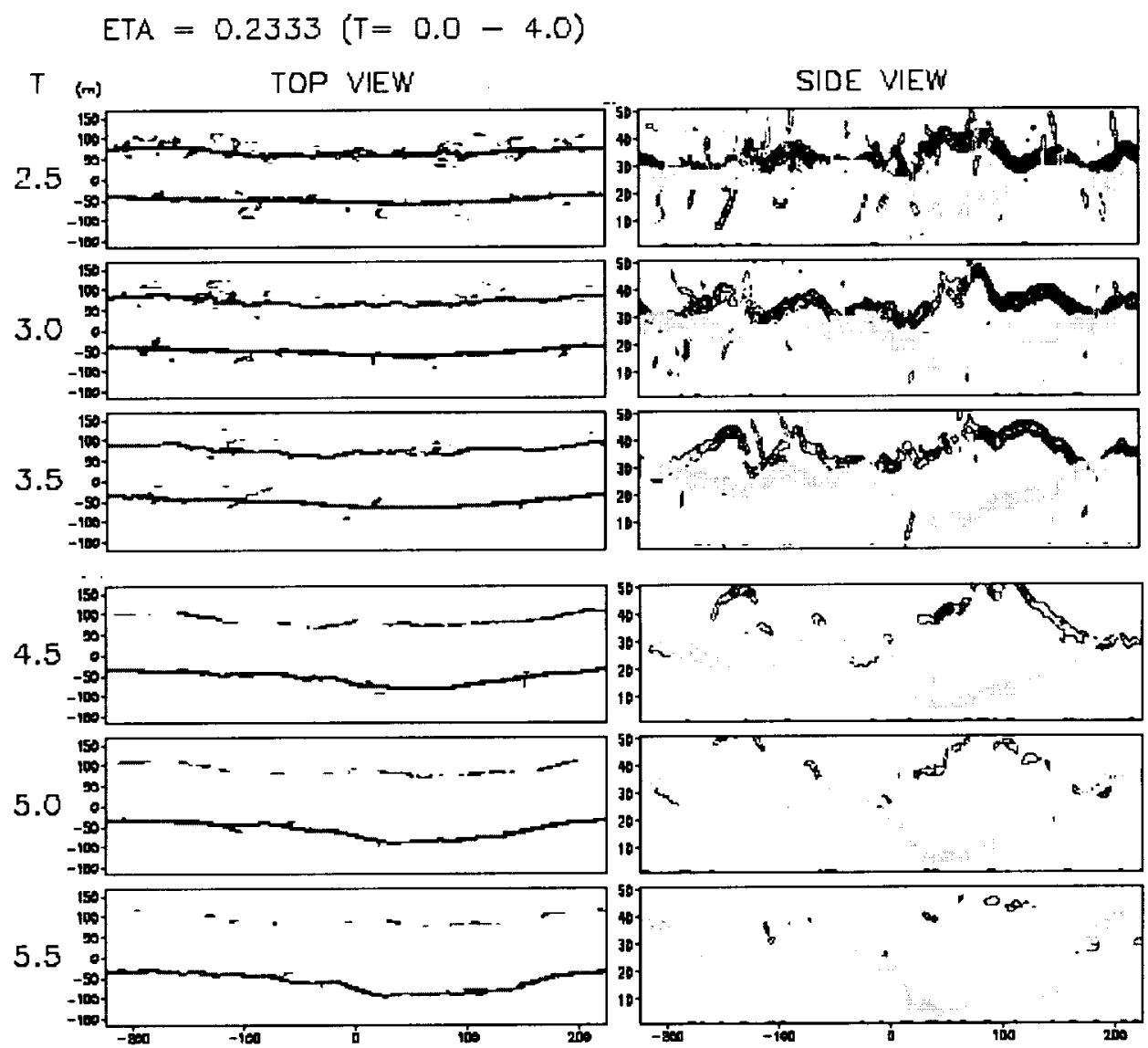

Figure 8. Same as Fig. 7, but for $\eta=0.233$. 
Results from these simulations showed obvious ground linking to occur when $\eta$ exceeded 0.3 (e.g. Fig 7). For lower values of nondimensional turbulence intensity, pronounced vertical oscillations developed without obvious linking between the wake vortex and its ground image (e.g. Fig. 8). In none of the cases (which are all initiated well within JGE) did lateral linking occur. Influence of the very weak crosswind shear is unknown (see Fig. Sb), but ground linking only occurred with the port vortex.

The visualization of the simulated wake vortex as it links with the ground is shown in Fig. 7. The port vortex becomes nearly vertical as it links with the ground just after $T=2$. For comparison. a photograph of an actual ground link is shown in Fig. 9.

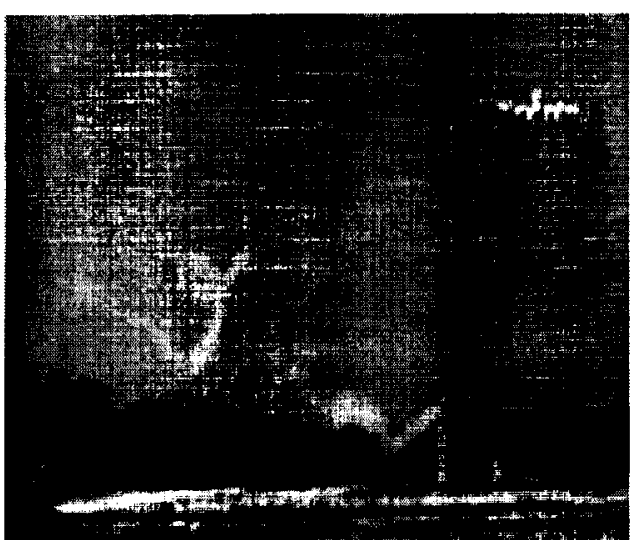

Figure 9. Observed case of ground linking (from NASA Langley photo archives, photograph L 90 02905)

Both port and starboard vortices in Fig. 7 rapidly dissipate after linking, becoming undistinguishable from background turbulence at $T=4$. Note that in the vicinity of where the ground connection is to occur, the starboard vortex is transported upward and the port vortex downward.

Figure 8 depicts the wake vortex system for a more moderate level of turbulence. Although an obvious link with the ground did not occur, a major portion of the port vortex remained parallel and very close to the ground. Again, note that the starboard vortex is at a relatively high altitude where the port vortex is closest to the ground.
A ground linking factor can be defined similarly to Crow's linking parameter ${ }^{14}$ as:

$$
\beta(1)=\frac{z_{\max }-z_{\min }}{z_{\max }+z_{\min }}
$$

where $z_{\max }$ and $z_{\min }$ are respectively, the maximum and minimum altitude of one of the vortices. Either the port or starboard vortex may be considered linked with its ground image when the linking factor exceeds 0.85 . Table 6 shows nondimensional times for $\beta$ to exceed both 0.75 and 0.85 as a function of nondimensional turbulence intensity. Also included is the linking time as predicted by Sarpkaya" ${ }^{17.34 .35}$ re-derivation of Crow and Bate's $\mathrm{s}^{32}$ theoretical formula for vortex lifespan in the free atmosphere. In our experiments, ground linking did not occur for $\eta<0.388$, although the vertical linking parameter did grow with time. Additional experiments were carried out for larger domain sizes assuming $\eta=0.233$, but little sensitivity was noted. Therefore, the linking time (or lack of linking) was not believed to be affected by our domain size. The influence of the crosswind shear on the suppression of linking for the lower turbulence levels is yet to be examined.

Table 6. Sensitivity of Ground Linking to Ambient Turbulence (port vortex).

\begin{tabular}{llll}
\hline$\eta$ & $\mathbf{T}_{\boldsymbol{\beta}=0.75}$ & $\mathbf{T}_{\boldsymbol{\beta}=\mathbf{0 . 8 5}}$ & $\mathbf{T}_{\text {Sarp }}$ \\
\hline 0.233 & 4.3 & $\cdots-.-$ & 2.4 \\
0.30 & 2.90 & ----- & 2.0 \\
0.388 & 1.90 & 2.10 & 1.71 \\
0.50 & 1.47 & 1.63 & 1.43 \\
0.75 & 0.98 & 1.14 & 1.11 \\
\hline
\end{tabular}

Figure 10 shows a comparison of the time to ground link with experimental data ${ }^{33}$ and theory ${ }^{32.34}$ for vortex linking in the free atmosphere. The three simulations that had ground linking are in close agreement with Sarpkaya's formula, ${ }^{34.35}$ even though the theory was developed for lateral vortex linking away from any ground influence 
Vortex Lifespan (time to link or burst) vs Turbulence Intensity

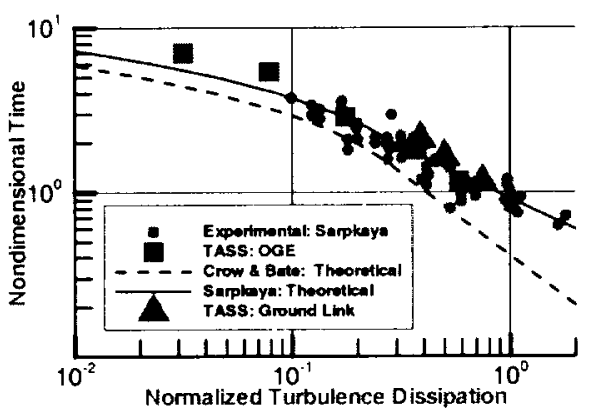

Figure 10. Linking time vs nondimensional turbulence intensity. Time of ground linking from LES given by triangles, all other data for lateral linking (or vortex bursting) out of ground effect (OGE).

\section{Summary and Conclusions}

A study of wake vortices initialized in-ground effect has been performed using a validated threedimensional LES model. The simulations, which included environmental turbulence, show strong decay of wake vortices following maximum penetration into IGE. Wake vortex linking with its ground image was simulated for moderate to strong values of atmospheric turbulence.

The primary conclusions of this study are:

1. Wake vortex transport and decay for IGE can be nondimensionalized with conventional parameters.

2. Appropriate normalization of parameters reduces wake vortex sensitivity to initial circulation, initial separation, and generating height.

3. Vortex decay for IGE has minor sensitivity to the ambient turbulence level.

4. Based on the LES results, a formula for IGE decay is proposed. This formula, with few dependent parameters, should be easy to incorporate in wake vortex prediction models.

5. Also based on the LES results, a model for lateral drift rate due to ground effect is proposed.

6. Both the decay and drift models show very good agreement with the LES sensitivity results.
7. Ground linking is influenced by the level of ambient turbulence. Similar to lateral linking in the free atmosphere, the time to ground link is a function of the nondimensional eddy dissipation. However, ground linking did not occur for moderate to weak levels of turbulence.

8. The increased lateral separation of vortices from ground effect can suppress lateral vortex linking.

Future work needs to address the sensitivity of crosswind shear to the onset vortex linking, both in and out of ground effect.

\section{Acknowledgements}

This research was sponsored by NASA's Terminal Area Productivity Program. One of the authors was funded under cooperative research grant NCC1-188. Numerical simulations were carried out on NASA and North Carolina Supercomputing Center supercomputers.

\section{Appendix - Sensitivity of Domain Size}

A number of experiments assume a truncated domain in the $\mathrm{x}$-direction, so as to reduce computation time, inhibit crow instability and simplify analysis of the experiments. The consequence of shortening the domain is evaluated here. Two simulations are carried out with everything identical except for a short domain: $\left(L_{x}, L_{y}, L_{z}=2.2 b_{o}, 10 b_{o}, 2.2 b_{o}\right)$, and a long domain $\left(L_{x}, L_{y}, L_{z}=12.2 b_{o}, 9.1 b_{o}, 2.2 b_{0}\right)$. The ambient turbulence level is assumed as $\eta=0.2349$ and each are resolved by the grid sizes listed in Table 3 . The ambient temperature and crosswind are from profiles observed at 20:09 UTC on 26 September 1997 at DFW (Fig. 5).

Comparison of the average circulations from the two simulations with different domain sizes is shown in Fig. A-I. Lidar observations from the $L-10 /]$ port vortex are also included. A comparison of lateral positions is shown in Fig. A-2. For this case, the figures indicate little sensitivity to domains size. 


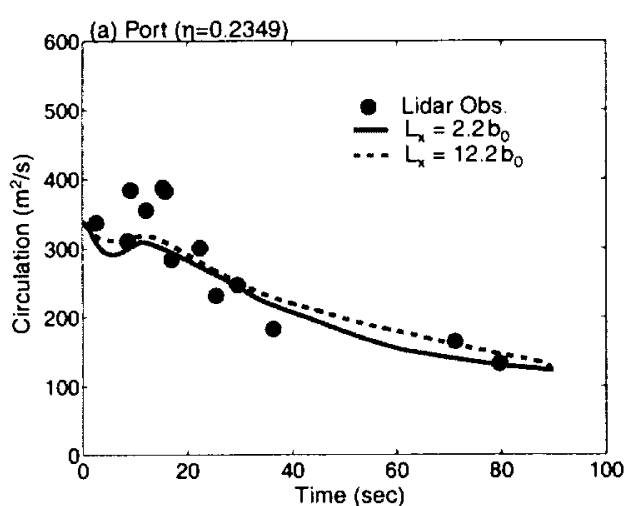

Figure A-1. Time evolution of the $5-15 \mathrm{~m}$ averaged circulation for port vortex from short domain $\left(L_{x}=2.2 b_{o}\right)$ and long domain $\left(L_{x}=12.2 b_{o}\right)$ simulations.

\section{References}

'Robins, R.E., Delisi, D.P. and Greene, G.C., "Development and Validation of a Wake Vortex Prediction Algorithm," $36^{\text {th }}$ Aerospace Sciences Meeting \& Exhibit, AIAA-98-0665, January 1998, 10 pp.

2Harvey, J.K., and Perty, F.J., "Flowfield Produced by Trailing Vortices in the Vicinity of the Ground," AlAA Journal, Vol. 9. No. 8, August 1971, pp. 16591660.

${ }^{3}$ Hallock. J.N.. "Wake Vortex Decay Near the Ground," AIAA $8^{\text {th }}$ Fluid and Plasma Dynamics Conf., Hartford, CN, AIAA-75-882, June 1975, 7 pp.

${ }^{4}$ Kopp, F., "Doppler Lidar Investigation of Wake Vortex Transport Between Closely Spaced Parallel Runways," AIAA J., Vol. 32, April 1994, pp.805-810.

"Hallock, J.N., and Burnham, D.C., "Decay Characteristics of Wake Vortices from Jet Transport Aircraft," $35^{\text {th }}$ Aerospace Sciences Meeting \& Exhibit, AIAA-97-0060, January 1997. 11 pp.

${ }^{6}$ Burnham, D C and Hallock, J N., "Measurements of Wake Vortices Interacting with the Ground." $36^{\text {th }}$ Aerospace Sciences Meeting \& Exhibit, AlAA-980593, January $1998,12 \mathrm{pp}$

${ }^{7}$ Rudis, R.P., Burnham, D.C., and Janota, P.. "Wake Vortex Decay Near the Ground under Conditions of Strong Stratification and Wind Shear," AGARD Fluid Dynamics Panel Symposium, Trondheim. Norway, AGARD CP-584, November, 1996, pp. 11-1 to $11-10$

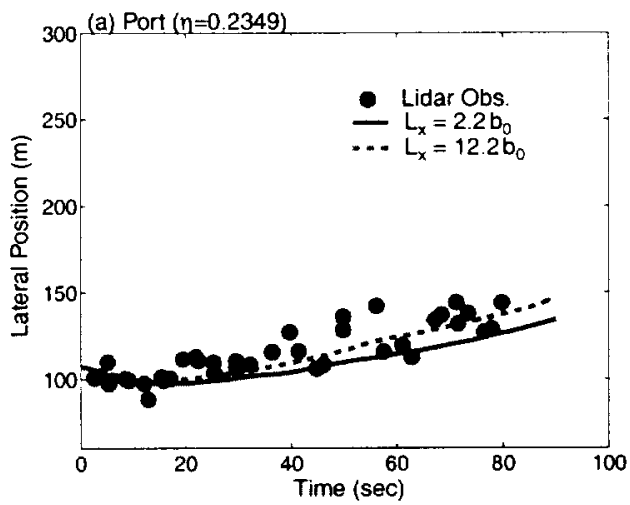

Figure A-2. Same as Fig. A-1, but for lateral position of port wortex.

${ }^{8}$ Schiling, V.K., "Motion and Decay of Trailing Vortices Within the Atmospheric Surface Layer," Beitr Phys. Almos., Vol. 65, May 1992, pp. 157-169.

"Robins, R.E. and Delisi, D.P., "Potential Hazard of Aircraft Wake Vortices in Ground Effect with Crosswind," Journal of Aircraft. Vol. 30, No. 2, MarchApril 1993, pp. 201-206.

${ }^{10}$ Zheng, Z.C., and Ash. R.L., "Study of Aircraft Wake Vortex Behavior Near the Ground," AIAA Journal, Vol. 34, No. 3, March 1996, pp. 580-589

"Corjon A., and Poinsot, T., "Behavior of Wake Vortices Near Ground," AIAA J., Vol. 35, No.5, May 1997, pp.849-855.

${ }^{12}$ Corjon, A., and Stoessel, A., "Three-Dimensional Instability of Wake Vortices Near the Ground," $28^{\text {th }}$ AIAA Fluid Dynamics Conf., Snowmass, CO, AlAA97-1782, June-July 1997, $14 \mathrm{pp}$

${ }^{13}$ Proctor, F.H., and Han, J., "Numerical Study of Wake Vortex Interaction with the Ground Using the Terminal Area Simulation System," $37^{\text {th }}$ Aerospace Sciences Meeting \& Exhibit, AIAA-99-0754, January 1999, 12 pp.

${ }^{14}$ Crow, S.C., "Stability Theory for a Pair of Trailing Vortices," AIAA Joumal, Vol. 8, No. 12, December 1970, pp. 2172-2179.

${ }^{15}$ Bisgood, P.L., Maltby, R.L., and Dee, F.W. "Some Work at the Royal Aircraft Establishment on the Behavior of Wake Vortices," In Aircraft Wake Turbulence and Its Detection, New York, Plenum, 1971.

${ }^{16}$ Spalart, P.R., "Airplane Trailing Vortices," Ann. Rev. Fluid Mech., Vol 30, 1998, pp. 107-138.

13 
${ }^{17}$ Sarpkaya, T., Robins, R.E., and Delisi, D.P., "Wake-Vortex Eddy-Dissipation Model Predictions Compared with Observations," $38^{\text {th }}$ Aerospace Sciences Meeting \& Exhibit, AlAA-2000-0625, January 2000.

${ }^{18}$ Hinton, D.A., "Aircraft Vortex Spacing System (AVOSS) Conceptual Design," NASA Tech. Memo No. 110184, August 1995, $27 \mathrm{pp}$.

${ }^{19}$ Perry, R. B., Hinton, D. A., and Stuever, R. A., "NASA Wake Vortex Research for Aircraft Spacing." $35^{\text {th }}$ Aerospace Sciences Meeting \& Exhibit, Reno, NV, AIAA 97-0057, January 1997, 9 pp.

${ }^{20}$ Hinton, D.A., Charnock, J.K., Bagwell, D.R., and Grigsby, D., "NASA Aircraft Vortex Spacing System Development Status," $37^{\text {th }}$ Aerospace Sciences Meeting \& Exhibit, Reno, NV, AIAA-99-0753, January 1999. $17 \mathrm{pp}$.

${ }^{21}$ Proctor, F.H., "The NASA-Langley Wake Vortex Modelling Effort in Support of an Operational Aircraft Spacing System," $36^{\text {th }}$ Aerospace Sciences Meeting \& Exhibit, AIAA-98-0589, January 1998, 19 pp.

"2NASA Langley Research Center, "Meteorological \& Wake Vortex Data Set, Dallas-Fort Worth International Airpor, September 15-October 3 , 1997," November 1998, Compact Disc

${ }^{23}$ Proctor, F.H., "The Terminal Area Simulation System. Volume 1: Theoretical Formulation," NASA Contractor Report 4046, DOT/FAA/PM-85/50, 1, April $1987,176 \mathrm{pp}$

${ }^{24}$ Proctor, F.H., "Numerical Simulation of Wake Vortices During the Idaho Falls and Memphis Field Programs," $14^{\text {th }}$ AIAA Applied Aerodynamics Conference, Proceedings, Part-I1, New Orleans, LA. AIAA-96-2496, June 1996, pp. 943-960.

${ }^{25}$ Switzer, G.F. "Validation Tests of TASS for Application to 3-D Vortex Simulations," NASA Contractor Report No. 4756, October 1996, 11 pp.

${ }^{26}$ Vincent, A. and Meneguzzi, M., "The Spatial Structure and Statistical Properties of Homogeneous Turbulence," Journal of Fluid Mechanics, Vol. 225, 1991, pp. 1-20.

${ }^{27}$ Kolmogorov, A.N., "The Local Structure of Turbulence in Incompressible Viscous Fluid for Very Large Reynolds Number," Dokl. Akad. Nauk SSSR, Vol. 30, 1941, pp 9-13.

${ }^{28}$ Han, J., Lin, Y.-L., Schowalter, D. G., Arya, S. P., and Proctor, F. H., "Large Eddy Simulation of Aircraft Wake Vortices within Homogeneous Turbulence: Crow Instability," AIAA Journal, Vol. 38, February, 2000.

${ }^{29} \mathrm{Han}$, J., Lin, Y.-L., Arya, S.P., and Proctor, F.H., "Numerical Study of Wake Vortex Decay and Descent within Homogeneous Turbulence," In press, AlAA Journal, Vol. 38, 2000.
${ }^{30}$ Burnham. D.C., and Hallock, J.N., "Chicago Monostatic Acoustic Vortex Sensing System, Volume IV: Wake Vortex Decay," Dept. of Transportation. Rep No. DOT/FAA/RD-79-103 IV, July 1982, 206 pp. (Available from NTIS]

${ }^{31}$ Hinton, D.A., and Tatnal, C.R., "A Candidate Wake Vortex Strength Definition for Application to the NASA Aircraft Vortex Spacing System (AVOSS)," NASA Tech. Memo. 110343, September 1997, 35 pp.

${ }^{32}$ Crow, S.C., and Bate, E.R., "Lifespan of Trailing Vortices in a Turbulent Atmosphere," $J$. Aircraft. Vol. 13, No. 7, 1976, pp. 476-482.

${ }^{33}$ Sarpkaya, T., and Daly, J.J., "Effect of Ambient Turbulence on Trailing Vortices," J. Aircraft, Vol. 24 No. 6, 1987, pp. 399-404.

${ }^{34}$ Sarpkaya, T., "Decay of Wake Vortices of Large Aircraft," AIAA J., Vol. 36, No. 9, 1998, 1671. 1679.

${ }^{35}$ Sarpkaya, T., "A New Model for Vortex Decay in the Atmosphere," $37^{\text {th }}$ Aerospace Sciences Meeting \& Exhibit, AIAA-99-0761, January 1999, 14 pp. 